\title{
Recent and future grand challenges in protein folding, misfolding, and degradation
}

\author{
Pierre Goloubinoff* \\ DBMV, Faculty of Biology and Medicine, University of Lausanne, Lausanne, Switzerland \\ *Correspondence: pierre.goloubinoff@unil.ch
}

Edited and reviewed by:

Matthias Peter Mayer, Ruprecht-Karls-Universität Heidelberg, Germany

Keywords: aggregation, proteopathies, crowding, molecular chaperones, proteases

Our general aim is to better understand the biophysical and biochemical principles that govern nascent or stress-destabilized proteins to unfold and re/fold to their native state or, alternatively, choose to misfold and aggregate into cytotoxic species, which in animals can cause degenerative diseases.

When a protein is first artificially unfolded by a chaotropic agent, it may thereafter spontaneously refold into its three dimensional native state without necessitating additional steric information from other macromolecules in the solution. Anfinsen (1973), thus masterfully demonstrated that the primary sequence of the amino acids contains sufficient information for polypeptides to reach their native functional three-dimensional structure. Yet, the yields of such in vitro protein refolding assays are typically low and a large fraction of the polypeptide precipitates into insoluble aggregates.

Living cells cannot accumulate high concentrations of chaotropic agents to specifically unfold potentially toxic aggregates, while leaving intact the surrounding native proteins. Instead they express molecular chaperones that can specifically recognize and bind with high-affinity to the misfolded polypeptides, which are to be unfolded into intermediates that can, thereafter, spontaneously refold into lowaffinity native products (Hinault et al., 2006; Priya et al., 2013). In the cell, heatstress can cause the partial transient opening of native proteins, typically at hinges between compact domains, and partially deconvoluted segments may then seek default stable conformers enriched with beta-sheet structures and with hydrophobic surfaces partially exposed to the aqueous phase (Natalello et al., 2013). Such intermediate species may then seek to reduce undesirable interactions with water and gain stability by forming strong cooperative hydrophobic hydrogen-bonds with other encountered intermediates, in a concentration-dependent oligomerization process generally dubbed "aggregation." The propensity of early-misfolded polypeptide species to form large insoluble aggregates depends on their initial concentration Dobson (2004) and also on the presence of small pre-formed aggregates that can act as seeds to the aggregation reaction (Gidalevitz et al., 2006).

In the last two decades, a major discovery has been that misfolded and aggregated conformers, even from "normal" non-toxic proteins, can become cytotoxic and infectious (Ben-Zvi and Goloubinoff, 2002). The proteotoxic effects of various pre-amyloid and proto-fibrillar forms of misfolded and aggregated proteins is a hallmark for many degenerative protein conformational diseases and is thought to be the main cause for late onset proteinopathies, such as Parkinson's, Alzheimer's, Amytrophic lateral sclerosis and diabetes types 2 (Luheshi and Dobson, 2009).

It is not clear why some early forms of aggregated proteins are toxic to mammalian cells, especially to neurons, where they apparently induce apoptosis. It is suggested that protofibrils may spontaneously form holes in membranes and disturb biological membranes in general (Lashuel and Lansbury, 2006). Moreover, they can lure other metastable proteins to misfold, aggregate, and become toxic, instead of spontaneously reverting after stress into harmless native proteins. Moreover, misfolded protein intermediates may stall the network of molecular chaperones and proteases that otherwise would effectively act as defenses of animal cells against proteotoxic conformers.

Even without aging and abiotic stresses, when polypeptides are synthetized in the ribosome, growing chains encounter the highly crowded environment of the cytoplasm, which is composed of up to $200 \mathrm{mg} / \mathrm{ml}$ proteins (Finka and Goloubinoff, 2013) and of ion and organic molecules in the hundreds millimolars range (Diamant et al., 2001). Cellular crowding is generally thought to favor misfolding and aggregation of unfolded polypeptides, such as new polypeptides emerging from the ribosome (Becker et al., 2013) or from an import pore. Yet, the presence of high concentrations of osmolytes or native proteins may stabilize already native proteins (Melo et al., 2010).

While emerging from the ribosome, intrinsically unfolded polypeptides may stay unfolded and, depending on the presence of osmolytes, pre-aggregated seeds or of stressful conditions, they may misfold and aggregate into toxic species as in the cases of $\alpha$-synuclein or tau (Luheshi and Dobson, 2009). Other de novo synthesized polypeptides may readily fold to the native state, as initially shown in vitro by Anfinsen in the simple case a monomeric enzyme. At variance, the concomitant or sequential assembly of multineric complexes may necessitate molecular chaperones to maintain near-native subunits in an assembly-competent state until other subunits are present to form functional oligomers. Polypeptides may also have to be prevented by cytoplasmic chaperones from completing folding while still in the wrong compartment and thus be maintained in a translocation-competent state poised to translocate through membrane 


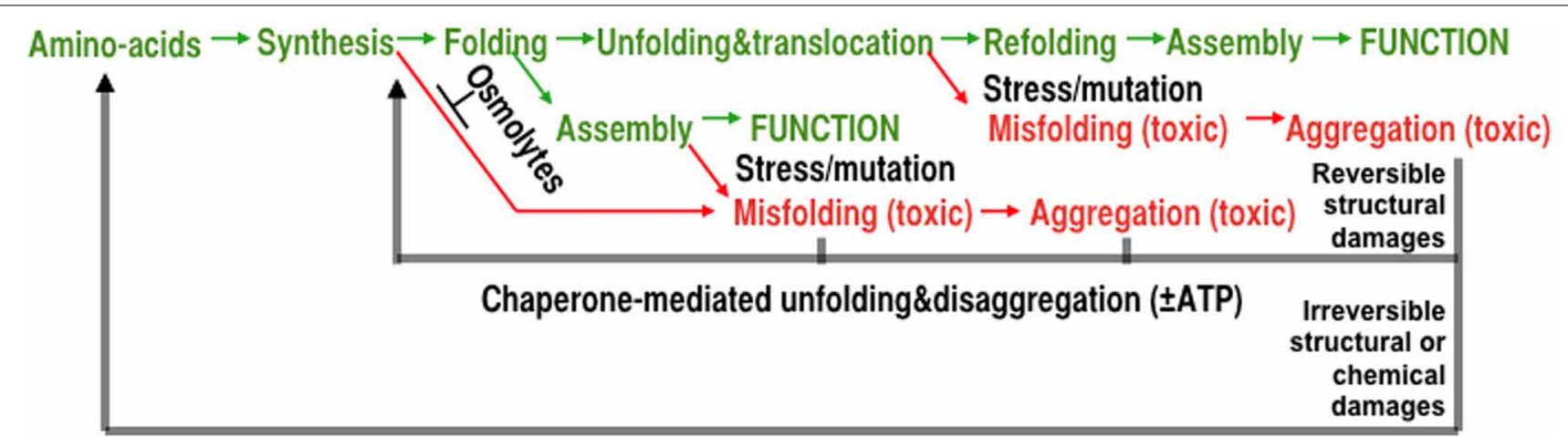

Chaperone/protease-mediated degradation ( \pm ATP)

FIGURE 1 | The various mechanisms of protein homeostasis. The maturation process of a polypeptide, from synthesis to the assembly of a native functional oligomer (green) is composed from delicate successive processes that can be affected by mutations, chemical modifications, and stress. Under stressful conditions, polypeptides may convert into misfolded and increasingly insoluble aggregates lacking specific biological activities.
Misfolded conformers can also induce misfolding of other metastable polypeptides, damage membranes, and in animal cells, cause apoptosis and tissue loss, aging and degenerative diseases. Whereas molecular chaperones can prevent and actively repair structural damages in reversibly damaged aggregates, proteases can degrade and recycle irreversibly damaged protein conformers. pores to the stroma of mitochondria, chloroplasts or the lumen of the endoplasmic reticulum (Figure 1. green paths).

Depending on the severity of various abiotic stresses, such as heat-shock, or owing to destabilizing chemical modifications or mutations, polypeptides may misfold and aggregate into potentially toxic conformers (Figure 1, red paths). Whereas osmolytes and so-called "holding" chaperones may prevent misfolding and aggregation, other chaperones can use the energy of ATP-hydrolysis to forcefully unfold and dissolve stable protein aggregates. Even without ATP hydrolysis, mere binding to particular molecular chaperones can unfold loosely misfolded species, which upon release, can refold to the native state. Thus, unfolding chaperones may provide renewed chances to already misfolded polypeptides to spontaneously refold into native functional proteins (Figure 1, black middle arrow). In case the aggregated conformers become irreversibly damaged structurally or chemically, chaperone-gated proteases can use the energy of ATP to forcefully unfold and solubilize aggregates into hydrolysable polypeptides, thereby generating free amino acids to re-synthetize new polypeptides (Figure 1, black lower arrow).

Frontiers in Molecular Biosciences, Protein folding, Misfolding, and Degradation publishes original biochemical research article addressing the processes of protein synthesis, folding, misfolding, translocation, refolding, assembly, and aggregation, as well as specific mechanisms by which chemical chaperones, molecular chaperones and proteases control cellular protein homeostasis. Of particular interest are physiological cellular processes, such as de novo folding and assembly of native complexes, which do not necessarily involve chaperones preventing or reverting protein aggregation. How may chaperones differentiate bound on-pathway nearnative subunits seeking to integrate native oligomers, from off-pathway oligomerorphan subunits that become rapidly degraded?

We are primarily interested in biochemical and molecular mechanistic studies addressing how chaperones and proteases use ATP hydrolysis to unfold, disaggregate and promote the native folding or the degradation of stably misfolded and aggregated proteins species and about the principles governing partitioning between the "rehabilitation" of misfolded proteins by unfolding and disaggregating chaperones, and "recycling" of misfolded proteins by unfolding proteases (Suraweera et al., 2012). We are also interested in proteomic biochemical and structural studies addressing the ways by which various misfolded and aggregated proteins accumulate in cells, and in the in vivo imaging of protein misfolding and amyloid accumulation in degenerative tissues and whole organisms.

\section{ACKNOWLEDGMENTS}

This project was financed by Swiss National Science Foundation 490 Grant 140512/1.

\section{REFERENCES}

Anfinsen, C. B. (1973). Studies on the principles that govern the folding of protein chains. Science 181, 223-230.

Becker, A. H., Oh, E., Weissman, J. S., Kramer, G., and Bukau, B. (2013). Selective ribosome profiling as a tool for studying the interaction of chaperones and targeting factors with nascent polypeptide chains and ribosomes. Nat. Protoc. 8, 2212-2239. doi: 10.1038/nprot.2013.133

Ben-Zvi, A. P., and Goloubinoff, P. (2002). Proteinaceous infectious behavior in nonpathogenic proteins is controlled by molecular chaperones. J. Biol. Chem. 277, 49422-49427. doi: 10.1074/jbc.M209163200

Diamant, S., Eliahu, N., Rosenthal, D., and Goloubinoff, P. (2001). Chemical chaperones regulate molecular chaperones in vitro and in cells under combined salt and heat stresses. J. Biol. Chem. 276, 39586-39591. doi: 10.1074/jbc.M103081200

Dobson, C. M. (2004). Principles of protein folding, misfolding and aggregation. Semin. Cell Dev. Biol. 15, 3-16. doi: 10.1016/j.semcdb.2003.12.008

Finka, A., and Goloubinoff, P. (2013). Proteomic data from human cell cultures refine mechanisms of chaperone-mediated protein homeostasis. Cell Stress Chaperones 18, 591-605. doi: 10.1007/s12192-013-0413-3

Gidalevitz, T., Ben-Zvi, A., Ho, K. H., Brignull, H. R., and Morimoto, R. I. (2006). Progressive disruption of cellular protein folding in models of polyglutamine diseases. Science 311, 1471-1474. doi: 10.1126/science.1124514 
Hinault, M. P., Ben-Zvi, A., and Goloubinoff, P. (2006). Chaperones and proteases - Cellular fold-controlling factors of proteins in neurodegenerative diseases and aging. J. Mol. Neurosci. 30, 249-265. doi: 10.1385/JMN: 30:3:249

Lashuel, H. A., and Lansbury, P. T. (2006). Are amyloid diseases caused by protein aggregates that mimic bacterial pore-forming toxins? Q. Rev. Biophys. 39, 167-201. doi: 10.1017/S0033583506004422

Luheshi, L. M., and Dobson, C. M. (2009). Bridging the gap: From protein misfolding to protein misfolding diseases. FEBS Lett. 583, 2581-2586. doi: 10.1016/j.febslet.2009.06.030

Melo, E. P., Estrela, N., Lopes, C., Matias, A. C., Tavares, E., and Ochoa-Mendes, V. (2010). Compacting proteins: pros and cons of osmolyte-induced folding. Curr. Protein Pept.
Sci. 11, 744-751. doi: 10.2174/13892031079 4557727

Natalello, N., Mattoo, R. U. H., Priya, S., Sharma, S. K., Goloubinoff, P., and Doglia, S. M. (2013). Biophysical characterization of two different stable misfolded monomeric polypeptides that are chaperone-amenable substrates. J. Mol. Biol. 425, 1158-1171. doi: 10.1016/j.jmb.2012. 12.025

Priya, S., Sharma, S. K., and Goloubinoff, P. (2013). Molecular chaperones as enzymes that catalytically unfold misfolded polypeptides. FEBS Lett. 587, 1981-1987. doi: 10.1016/j.febslet.2013. 05.014

Suraweera, A., Münch, C., Hanssum, A., and Bertolotti, A. (2012). Failure of amino acid homeostasis causes cell death following proteasome inhibition. Mol. Cell 48, 242-253. doi: 10.1016/j.molcel.2012.08.003
Received: 18 February 2014; accepted: 05 March 2014; published online: 27 March 2014.

Citation: Goloubinoff P (2014) Recent and future grand challenges in protein folding, misfolding, and degradation. Front. Mol. Biosci. 1:1. doi: 10.3389/fmolb. 2014.00001

This article was submitted to Protein Folding, Misfolding and Degradation, a section of the journal Frontiers in Molecular Biosciences.

Copyright (c) 2014 Goloubinoff. This is an open-access article distributed under the terms of the Creative Commons Attribution License (CC BY). The use, distribution or reproduction in other forums is permitted, provided the original author(s) or licensor are credited and that the original publication in this journal is cited, in accordance with accepted academic practice. No use, distribution or reproduction is permitted which does not comply with these terms. 